\title{
IMPLEMENTAÇÃO DE UM LABORATÓRIO DE RECURSOS PEDAGÓGICOS INCLUSIVOS - LARPI
}

\section{IMPLEMENTATION OF AN INCLUSIVE PEDAGOGICAL RESOURCES LABORATORY - LARPI}

\author{
VIEIRA, Júlia Maria Alves ${ }^{1}$ \\ QUINTANILHA, Beatriz Almeida ${ }^{2}$ \\ GUTHIERREZ, Carla Cordeiro Marçal $y^{3}$
}

\begin{abstract}
RESUMO
O presente artigo relata a experiência da implementação e organização de um Laboratório de Recursos Pedagógicos Inclusivos - LARPI no Instituto de Aplicação Fernando Rodrigues da Silveira, através de um Projeto de Estágio Interno Complementar. O LARPI foi criado em 2018 e consta com um acervo organizado com 38 produtos construídos e disponíveis para todos os estudantes do Departamento do Ensino Fundamental. Esses produtos são construídos a partir dos conteúdos trabalhados em sala de aula e visam atender e auxiliar, sobretudo, os estudantes com deficiência.
\end{abstract}

Palavras-chave: Educação Inclusiva; Recursos Pedagógicos Inclusivos; Comunicação Alternativa.

\section{ABSTRACT}

This article reports the experience of implementing and organizing an Inclusive Pedagogical Resources Laboratory - LARPI at the Fernando Rodrigues da Silveira Application Institute, through a Complementary Internal Internship Project. LARPI was created in 2018 and has an organized collection of 38 products built and available to all students in the Department of Elementary Education. These products are built from the contents worked in the classroom and aim to assist and assist, especially, disable students.

\footnotetext{
${ }^{1}$ Universidade do Estado do Rio de Janeiro (UERJ) / Projeto LARPI - Estágio Interno Complementar. Rio de Janeiro, RJ, Brasil. ORCID: https://orcid.org/0000-0001-9327-9060 e-mail: juliamalvesv@gmail.com

2 Universidade do Estado do Rio de Janeiro (UERJ) / Projeto de Extensão Universitária "Formação Continuada de Professores no Uso da Comunicação Alternativa". Rio de Janeiro, RJ, Brasil. ORCID: https://orcid.org/0000-0002-3588-4737 e-mail: quintanilhabia@gmail.com

3 Universidade do Estado do Rio de Janeiro (UERJ) / Instituto de Aplicação Fernando Rodrigues da Silveira (CAp-UERJ) / Departamento do Ensino Fundamental / Atendimento Educacional Especializado - AEE. Rio de Janeiro, RJ, Brasil. ORCID: https://orcid.org/0000-0001-7402-9365 e-mail: carlacordeiromarcal@gmail.com
} 
DOI: $10.12957 / \mathrm{e}-\mathrm{mosaicos} .2020 .51363$

KeYwORDS: Inclusive Education; Inclusive Pedagogical Resources; Alternative Communication.

\section{INTRODUÇÃO}

Assegurar a inclusão de estudantes com deficiências, transtornos globais do desenvolvimento e altas habilidades/superdotação é cumprir com a Política Nacional de Educação Especial na perspectiva da Educação Inclusiva (BRASIL, 2008) e mais que isso, é contribuir e garantir a todos o acesso ao ensino regular com participação, aprendizagem e continuidade nos níveis mais elevados do ensino.

Neste artigo, relata-se a implementação de um Laboratório de Recursos Pedagógicos Inclusivos - LARPI, no ano de 2018, no Instituto de Aplicação Fernando Rodrigues da Silveira, parte de um projeto de Estágio Interno Complementar que surgiu após um projeto de construção de recursos pedagógicos. Diante da criação de diversos produtos para proporcionar o aprendizado dos estudantes público-alvo da Educação Especial, a organização e sistematização desses produtos passaram a ser necessárias. A catalogação do acervo possibilita identificar os produtos criados, materiais utilizados para confecção e o ano de escolaridade atendido.

O LARPI se desenvolve no departamento de ensino fundamental atendendo estudantes público-alvo da Educação Especial, sobretudo com Transtorno do Espectro Autista - TEA, podendo atender estudantes de outros segmentos.

Os produtos são criados pelo projeto "Recursos De Tecnologia Assistiva E Comunicação Alternativa E Ampliada Na Perspectiva Da Educação Inclusiva" a partir da necessidade dos estudantes com deficiências e/ou transtornos e visam trazer acessibilidade aos conteúdos pedagógicos, aprendizado e desenvolvimento. Muitos desses produtos estão articulados a recursos de Tecnologia Assistiva (TA). Vale dizer, que TA agrupa dispositivos, técnicas e recursos que contribuem para proporcionar e ampliar algumas habilidades funcionais de pessoas com deficiências. Esta expressão pode dar a impressão de que são recursos de elevada sofisticação tecnológica, porém, a maioria deles é simples, como projetar um assento e um encosto na cadeira que permitam estabilidade postural e favoreçam o uso funcional das mãos. (BERSCH, 2007). Segundo Oliveira e Machado (2007), são ajustes realizados no currículo, para que se torne apropriado ao acolhimento das diversidades do alunado, sendo verdadeiramente um currículo inclusivo. Essas adaptações envolvem modificações organizativas. Isso permite 0 atendimento às necessidades educativas de todos os estudantes nos objetivos e conteúdos, nas metodologias e organização didática, na organização do tempo e na filosofia e estratégias de avaliação em relação à construção do conhecimento.

No decorrer da implementação do LARPI, percebeu-se o quanto o levantamento bibliográfico acerca das temáticas e fundamentações teóricas proporciona a elaboração de recursos e serviços de TA e Comunicação Alternativa e Ampliada (CAA) e possibilita a vivência em sala de aula e a aplicação de ensinamentos estudados a estudantes com as mais variadas capacidades. 
DOI: $10.12957 / \mathrm{e}-\mathrm{mosaicos} .2020 .51363$

\section{MetOdologia}

Para a implementação do LARPI, criou-se um acervo para organização e sistematização dos produtos. Além disso, faz-se necessária a observação semanal dos estudantes em sala de aula para compreender as dificuldades em relação aos conteúdos aplicados e desafios enfrentados no cotidiano escolar, considerando as dificuldades motoras, cognitivas e sociais, para que sejam trabalhadas no atendimento educacional especializado. Os produtos abrangem o conteúdo visto em sala de aula, para que o estudante com deficiência cumpra com o pedagógico, aprenda e se desenvolva em seu ano de escolaridade.

Os produtos podem ser utilizados por todos estudantes e são, em grande parte, produzidos com materiais acessíveis em sala de aula, como papel, cartolina, tesoura, cola, material para escrita. Quando há a necessidade, utiliza-se recursos exteriores como a impressão em gráfica e plastificação para melhor conservação do produto.

Ao confeccionar materiais para ampliar a comunicação, a exemplo de cartões de CAA, as pesquisas e busca de pictogramas são feitas através de páginas na internet, como a plataforma $A R A S A A C^{4}$ e 0 aplicativo Picto $4 M e^{5}$. Em ambos são encontradas imagens para criação de pranchas e cartões de CAA.

Os produtos são então categorizados de acordo com o conteúdo e ano de escolaridade através de um acervo digital criado com tabela enumerada no excel conforme a criação dos produtos, atualizada com novas produções e há a elaboração de um laboratório físico para armazenamento destes materiais.

\section{Resultados e Discussões}

Em maio de 2020, a produção e categorização dos materiais conta com 38 (trinta e oito) produtos abrangendo jogos lúdicos, como de tabelas de multiplicação; jogos da memória baseados em textos trabalhados em sala de aula; cartões de CAA, podendo ser usados para ilustrar ações cotidianas realizadas dentro e fora do ambiente escolar, servindo de apoio e estímulo para comunicação e interação entre os estudantes sem fala funcional, suas professoras e colegas.

A elaboração e organização desses produtos em laboratório físico com acervo digital permitem o acesso de estudantes e professores quando seu uso se faz necessário.

É preciso um aprimorar a qualidade do ensino e trabalhar com princípios educacionais válidos para todos, incluindo os estudantes com deficiência. Segundo Marçal-Guthierrez, Paula, Quintanilha e Ribeiro (2019, p. 139):

Para que haja respeito à diversidade na escola, é necessário que todos

\footnotetext{
${ }^{4}$ Sistema ARASAAC - Trata-se de um conjunto em torno de 15.000 pictogramas, com licença livre, que podem ser usados para a criação de diversos tipos de materiais pedagógicos e tabelas de comunicação. ${ }^{5}$ Aplicativo online para criação de pranchas de comunicação pictográficas para pessoas com deficiência.
} 
DOI: $10.12957 /$ e-mosaicos.2020.51363

sejam reconhecidos como iguais em dignidade e em direito, porém sem deixar de considerar as inúmeras formas de diferenciação que existem entre os indivíduos e os grupos. Devemos fornecer o apoio e os recursos necessários para que não haja tanta "assimetria", desigualdade nas oportunidades e no acesso aos recursos.

Os desafios enfrentados na escola e no convívio social, gerados pelas diferenças entre os indivíduos, devem ser minimizados ao máximo para que não ocorra segregação e discriminação com os estudantes, em especial aos considerados "diferentes". As necessidades de aprendizagem e comunicação são pessoais e é preciso, então, uma visão individualizada quanto a essas questões para cada estudante.

Alguns produtos do LARPI:

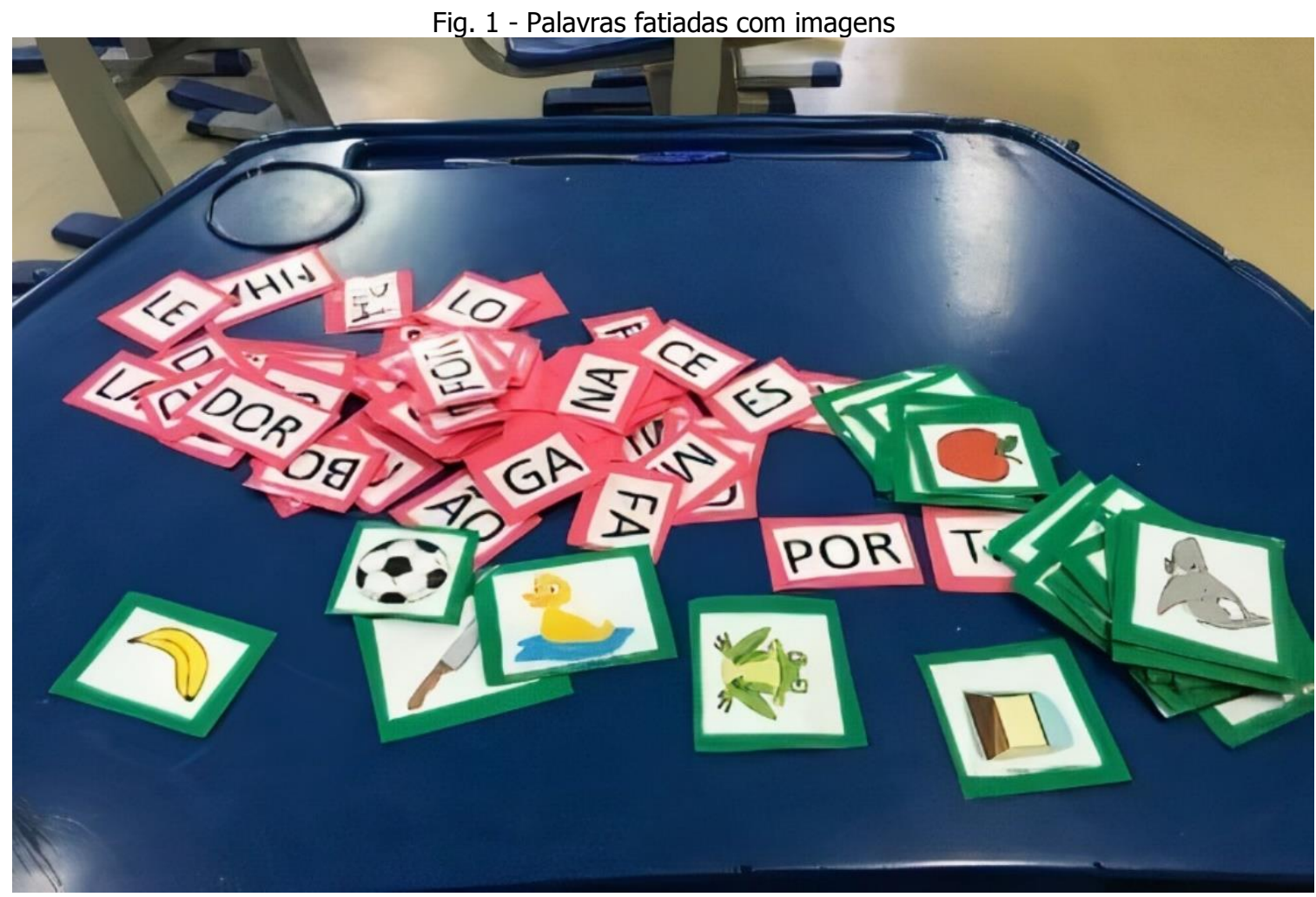

Fonte: Acervo LARPI 
DOI: $10.12957 / \mathrm{e}-\mathrm{mosaicos} .2020 .51363$

Fig. 2 - Pranchas móveis com cartões de CAA

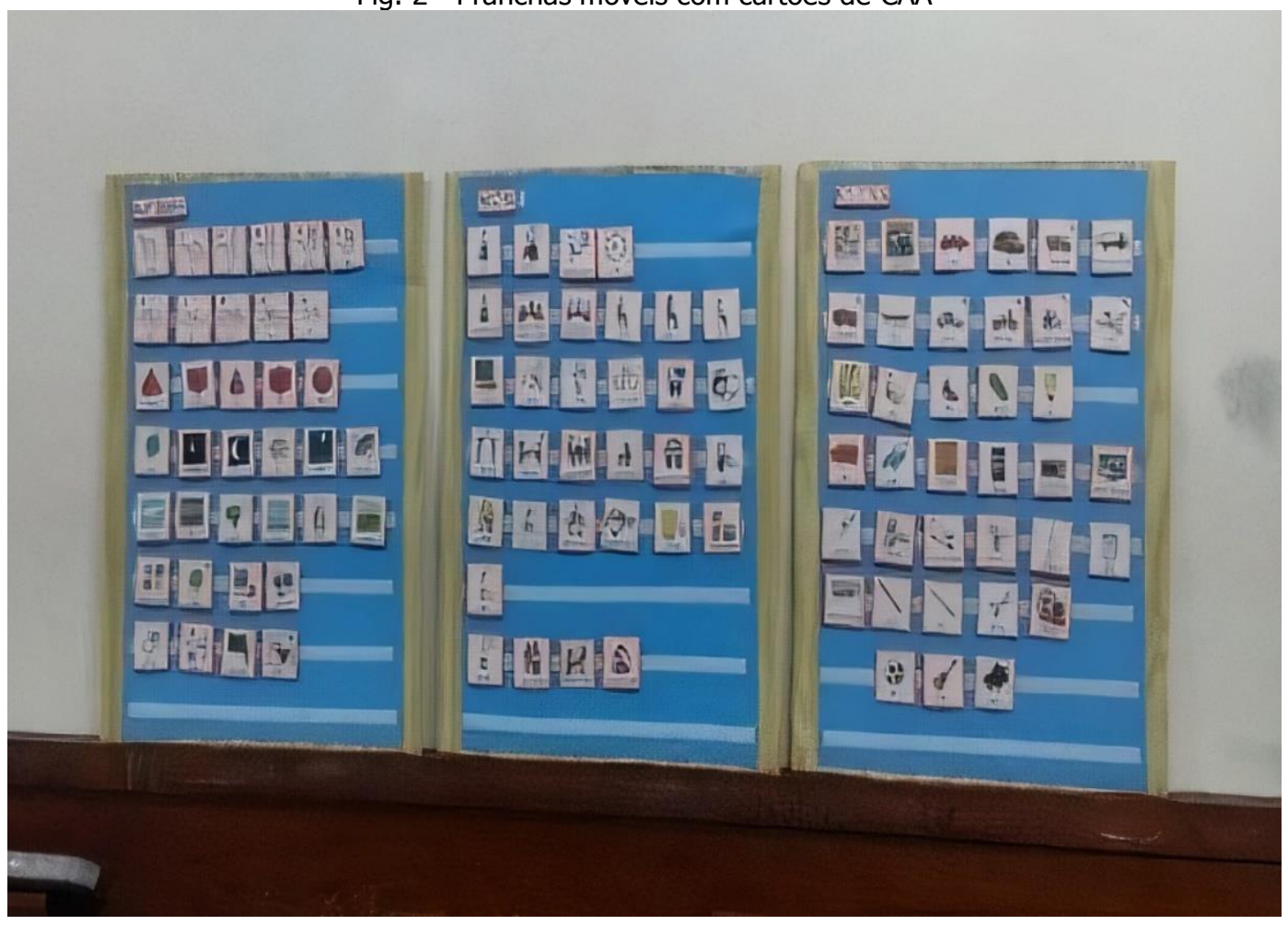

Fonte: Acervo LARPI

Fig. 3 - Tabuleiro da Multiplicação

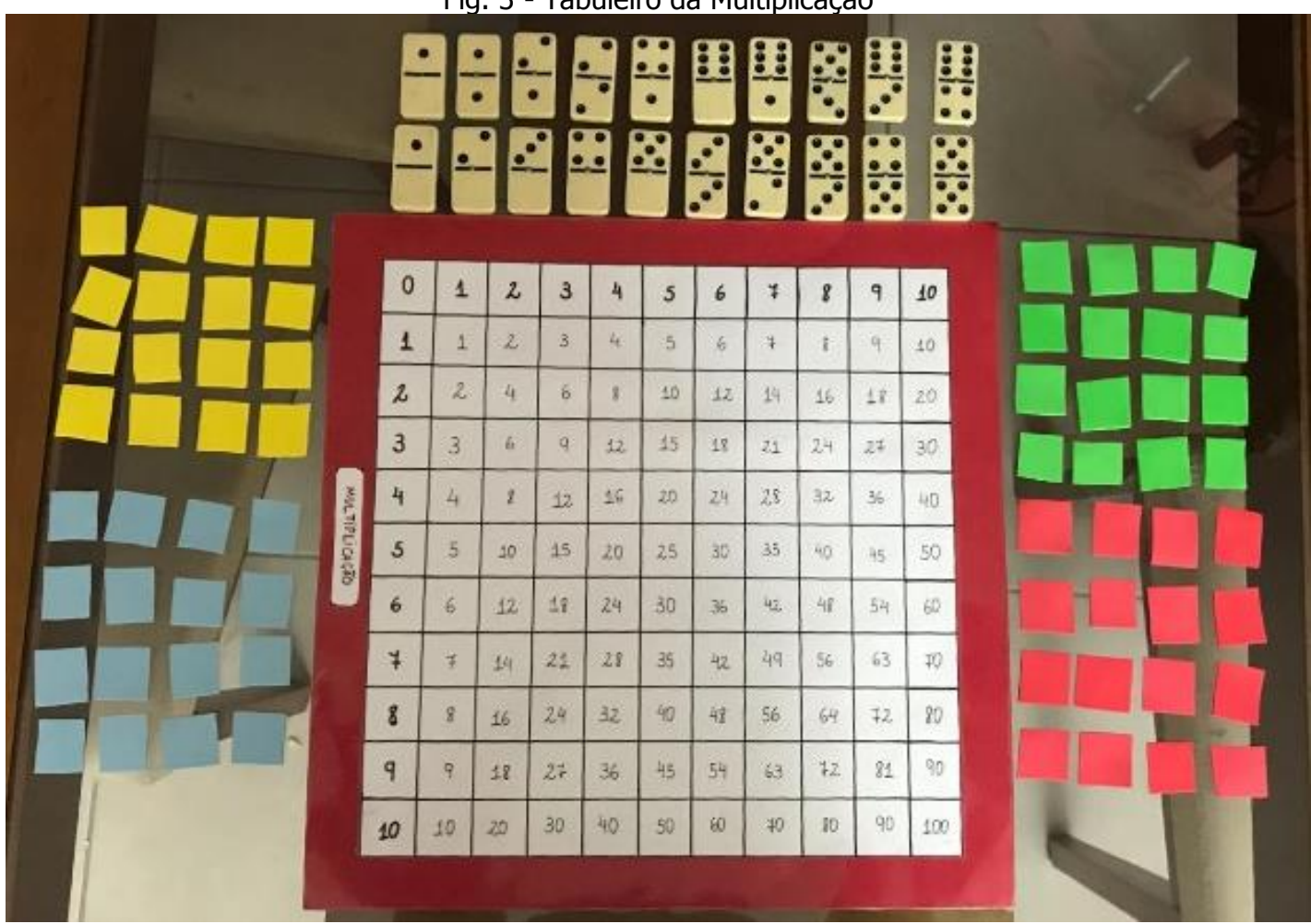

Fonte: Acervo LARPI 


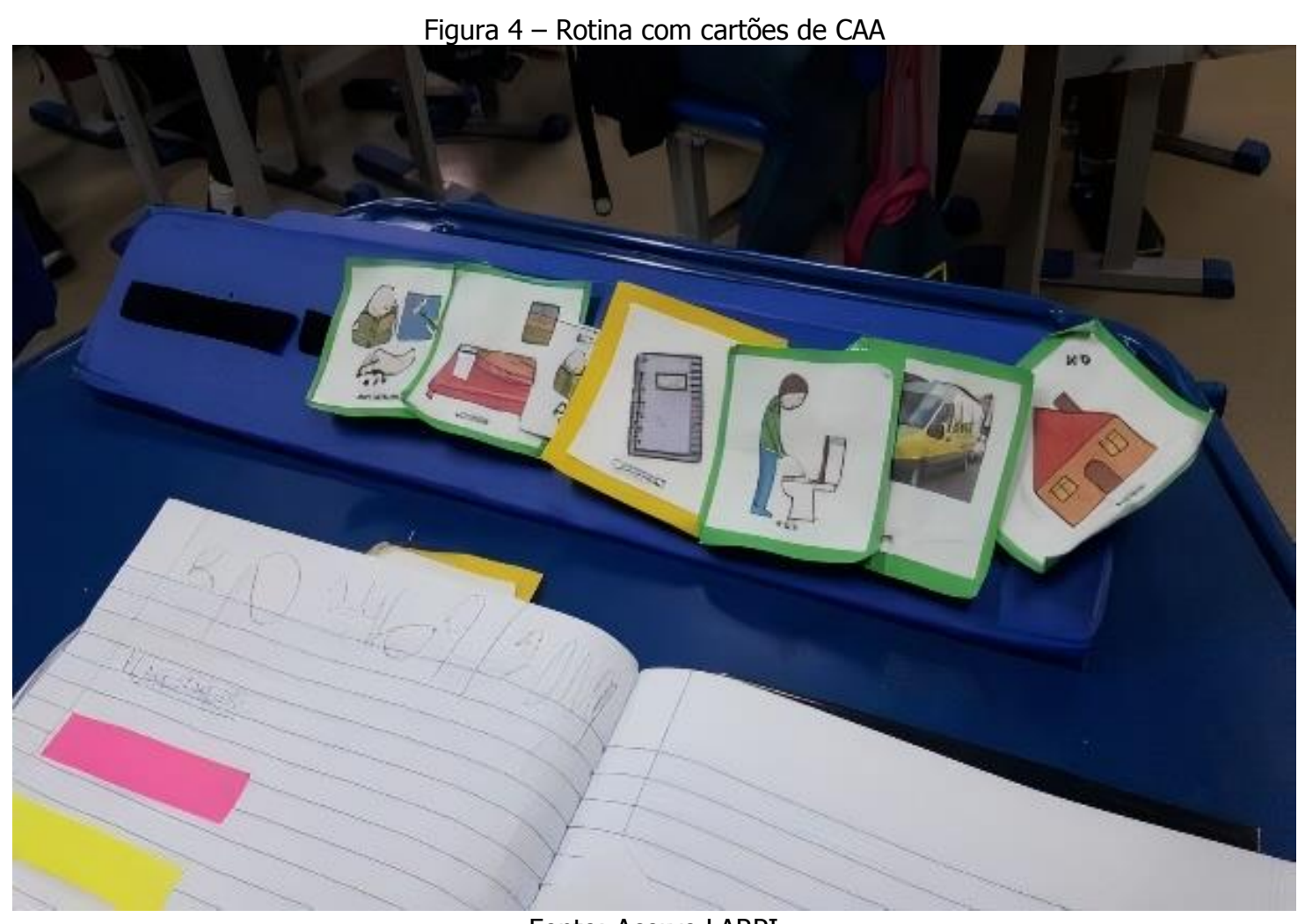

Fonte: Acervo LARPI

\section{CONSIDERAÇõES FINAIS}

O LARPI tem proporcionado a organização dos produtos criados no cotidiano da escola e, sobretudo, contribuído na eliminação das barreiras do currículo e da aprendizagem.

A inclusão escolar é um dos pilares da elaboração do currículo e propõe uma política de justiça social, buscando atender as necessidades educacionais de todos os estudantes em prol de uma educação de qualidade para todos. Ao invés de focalizar a deficiência, enfatiza o ensino e a escola, bem como as formas e as condições de aprendizagem.

Busca-se uma educação inclusiva e isso significa uma busca constante por uma sociedade democrática e justa.

\section{REFERÊNCIAS}

BERCSH, R. C. R.; PELOSI, M. B. Portal para ajudas técnicas. Tecnologia Assistiva: recursos de acessibilidade ao computador. Brasília: MEC/SEESP, 2007.

BRASIL. Política Nacional de Educação Especial na perspectiva da Educação Inclusiva. Brasília, janeiro de 2008. 
DOI: $10.12957 / \mathrm{e}-\mathrm{mosaicos} .2020 .51363$

OLIVEIRA, E. e MACHADO, K.S. Adaptações curriculares: caminho para uma Educação Inclusiva. In: GLAT, Rosana. Educação Inclusiva: cultura e cotidiano escolar. Rio de Janeiro: 7Letras, 2007.

PAULA, M. C. B. L.; MARÇAL, C. C; QUINTANILHA, B. A.; RIBEIRO, Apanache. Diferenciação pedagógica, atendimento educacional especializado e o ensino colaborativo na perspectiva inclusiva. In: MARÇAL-GUTHIERREZ, C.C; PRATA, Juliana de Moraes e CRUZ, Mara Monteiro. Práticas e perspectivas de ensino colaborativo Rio de Janeiro: CAP/UERJ, 2019.

Recebido em 28 de maio de 2019

Aceito em 28 de setembro de 2020

A e-Mosaicos Revista Multidisciplinar de Ensino, Pesquisa, Extensão e Cultura do Instituto de Aplicação Fernando Rodrigues da Silveira (CAp-UERJ) está disponibilizada sob uma Licença Creative Commons - Atribuição-NãoComercial 4.0 Internacional.

Os direitos autorais de todos os trabalhos publicados na revista pertencem ao(s) seu(s) autor(es) e coautor(es), com o direito de primeira publicação cedido à e-Mosaicos.

Os artigos publicados são de acesso público, de uso gratuito, com atribuição de autoria obrigatória, para aplicações de finalidade educacional e não-comercial, de acordo com o modelo de licenciamento Creative Commons adotado pela revista. 